\title{
Erratum: Long-range correlations in the electric signals that precede rupture [Phys. Rev. E 66, 011902 (2002)]
}

P. A. Varotsos, * N. V. Sarlis, and E. S. Skordas

(Received 21 October 2008; published 7 November 2008)

DOI: 10.1103/PhysRevE.78.059902

PACS number(s): 87.17.-d, 05.40.-a, 99.10.Cd

It was brought to our attention that there is a typographical error in Ref. [22] and that Ref. [12] is not complete. The correct Refs. [12] and [22] should read as follows:

[12] P. Varotsos, Acta Geophys. Pol. 49, 1 (2001); P. Varotsos, K. Alexopoulos, and K. Nomicos, Phys. Status Solidi B 111, 581 (1982); see also P. Varotsos and K. Alexopoulos, J. Phys. Chem. Solids 41, 443 (1980); 42, 409 (1981).

[22] P. Varotsos, N. Sarlis, and E. Skordas, Practica of the Athens Academy 76, 294 (2001).

\footnotetext{
*pvaro@otenet.gr
} 